\title{
TEATRO Y PEDAGOGÍA. RECONTEXTUALIZACIÓN CURRICULAR DE UN PROGRAMA DE ARTES ESCENICAS ${ }^{1}$
}

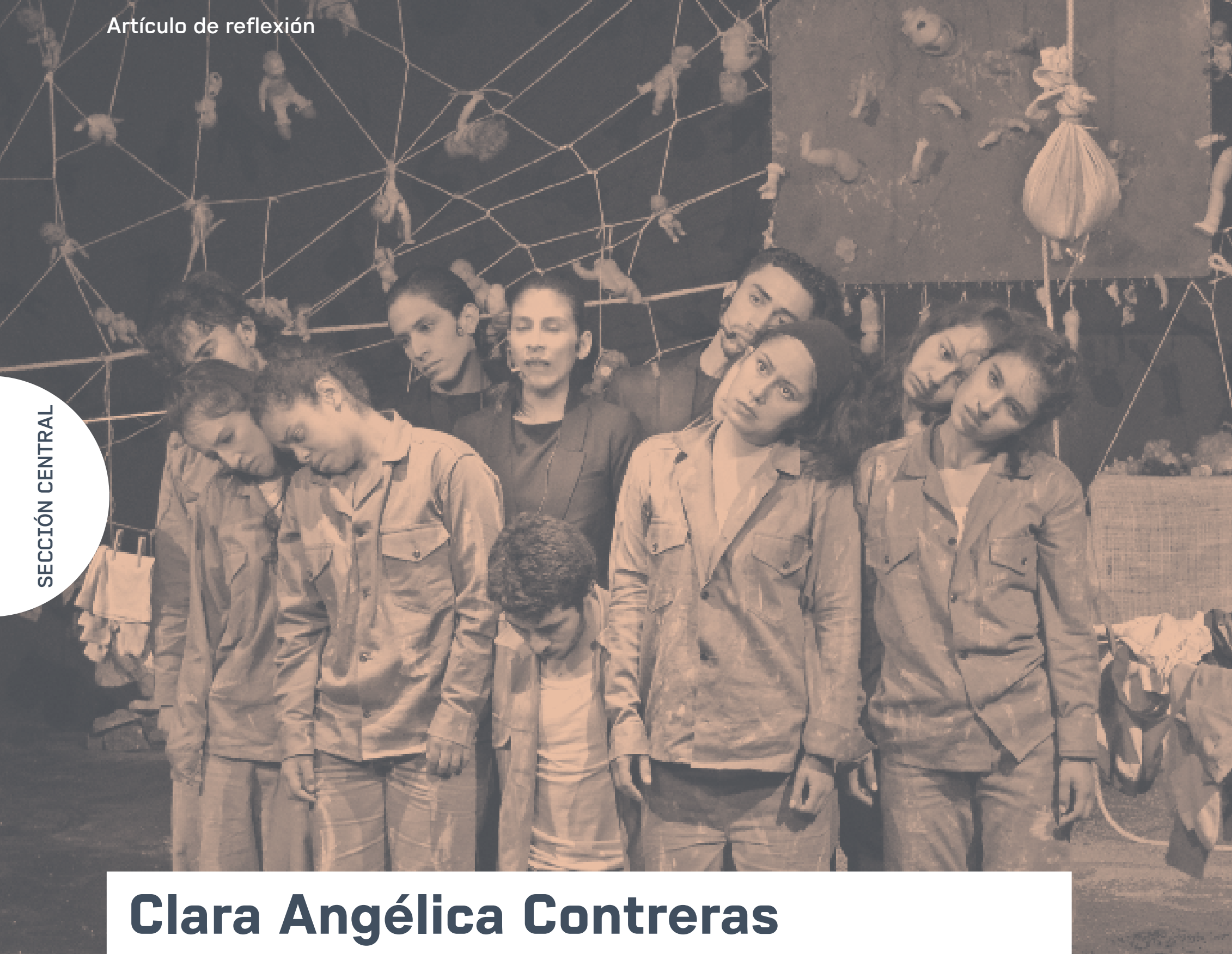

Secretaria de Educación Nacional / claris_2006@hotmail.com

Maestra de Artes Escénicas con énfasis en Dirección del programa de Artes Escénicas, Facultad de ArtesASAB, U.D.F.J.C. Magíster en Educación en la línea de investigación Educación Superior de la Universidad Pedagógica Nacional. Actualmente se desempeña como docente de teatro de la Secretaria de Educación de Bogotá; forma parte del grupo de investigación "Estudios de la voz y la palabra"; pertenece al grupo Vendimia Teatro, donde participa como directora y actriz. Sus inquietudes investigativas se enmarcan en el campo de la pedagogía teatral, la educación formal, currículo y evaluación en programas de artes escénicas. 
CONTRERAS C, (2014) Teatro y pedagogía. Recontextualización curricular de un programa de artes escénicas. Calle14,8 (12), 80-94.

\title{
RESUMEN
}

Tomando como telón de fondo a la antigua Academia Superior de Artes de Bogotá (actual Facultad de Artes - ASAB de la Universidad Distrital Francisco José de Caldas) y específicamente el programa de artes escénicas en sus énfasis de actuación y dirección, se hace una reflexión en torno a las consecuencias de las recontextualizaciones curriculares en un programa de esa índole. Es importante entender estos cambios en el contexto de la globalización, una era en que el mercado determina no solo las relaciones, sino las experiencias humanas. Para satisfacer las demandas sociales capitalistas la universidad se ha entregado a una actualización de sus orientaciones, principios y dinámicas, y a la vertiginosa labor que requiere cumplir con los requisitos necesarios para mantener su prestigio en el campo académico.

\section{PALABRAS CLAVES}

Facultad de Artes ASAB, programa curricular, artes escénicas, actuación, dirección, globalización

\section{THEATRE AND PEDAGOGY. CURRICULAR RECONTEXTUALIZATION OF A PERFORMING ARTS PROGRAM}

\begin{abstract}
With the backdrop of the old Academia Superior de Artes de Bogotá (now the Faculty of Arts ASAB of Francisco José de Caldas District University) and specifically of the Performing Arts program in its emphases in acting and directing, the article reflects on the consequences of curricular recontextualizations in a program of this kind. It is important to understand these changes in the context of globalization, an era in which the market determines not only relationships, but human experiences. To meet the social demands of capitalism, the university has updated its orientation, principles and dynamics, and has taken on the dizzying task of fulfilling the requirements that are necessary to maintain its prestige in the academic field.
\end{abstract}

\section{KEYWORDS}

ASAB Faculty of Arts, curriculum, performing arts, acting, directing, globalization

\section{THÉÂTRE ET PÉDAGOGIE. RE-CONTEXTUALISATION D'UN PROGRAMME CURRICULAIRE D'ARTS DE LA SCÈNE}

\section{RÉSUMÉ}

Prenant comme toile de fond l'Antique Académie Supérieure des Beaux-Arts de Bogotá (actuelle Faculté des Beaux-Arts - ASAB de I'Université du District Francisco José de Caldas) et en particulier le programme des arts du spectacle dans sa mise en valeur de l'art théâtral et de mise en scène, une réflexion est faite sur les conséquences des re-contextualisations curriculaires dans un tel programme. Il est important de comprendre ces changements dans le contexte de la mondialisation, une époque où le marché détermine non seulement les relations, mais aussi les expériences humaines. Pour répondre aux demandes de la société capitaliste l'université s'est 
livrée à une mise à jour de ses orientations, de ses principes et dynamiques, ainsi qu'à la tâche vertigineuse que demande l'accomplissement des conditions nécessaires pour maintenir son prestige dans le domaine académique.

\section{MOTS CLÉS}

Faculté des Beaux-Arts ASAB, programme curriculaire, arts de la scène, art théâtrale, mise en scène, globalisation

\section{TEATRO E PEDAGOGIA. RECONTEXTUALIZAÇÃO CURRICULAR DE UM PROGRAMA DE ARTES CÊNICAS}

\section{RESUMO}

Tomando como pano de fundo a antiga acadêmica superior de artes de Bogotá (atual Faculdade de Artes ASAB da Universidade Distrital Francisco José de Caldas) e especificamente o programa de artes cênicas em suas ênfases de atuação e direção, faz-se uma reflexão em torno das consequências das recontextualizações curriculares num programa dessa índole. É importante entender estes câmbios no contexto da globalização, uma era em que o mercado determina não só as relações, senão as experiências humanas. Para satisfazer às demandas sociais capitalistas, a universidade se entregou a uma atualização de suas orientações, princípios e dinâmicas e à vertiginosa tarefa que requer cumprir com os requisitos necessários para manter seu prestigio no campo acadêmico.

\section{PALAVRAS-CHAVE:}

Faculdade de Artes ASAB, programa curricular, artes cênicas, atuação, direção, globalização.

\section{IACHACHISKATA KAUACHIRINGAPA}

\section{SUGLLAPI MAILLA KILKAILLATA}

Nugpamandata kauaridiru atun iachaikui iuaita kauachispa, Bogotamanta Kunaura kanmi Atun iuiata kawachidiru. ASAB Francisco José de Caldas Distrital Universidadmanda allillapa, kai iachaikuikuna. Rimaspa, kauachispa apachispa sumami iuaikuna tia, Allim ka iachangapa imasami tukui trukarispa ririku. Kaikuna sug karkami katuipi randiipi. Mana chillapi kanmi, imasata iuia runakuna iachaikungapa manak sugkunalla. Iachaspa chiwanmi chi atun iachachigmanda, wasikunamanta kallaririrka tukuikunata suglla iuiaiwa Iachachingapa ñugpamandata kuiuchispa ñugpalla imasami ka chasa allilla, iachachinakungapa.

\section{IMA SUTI RIMAI SIMI}

Allilla iachaikudiru ASAB, imasa iachaichingapa, iachaskakunata kawachingapa, masama, tukuipi. 
La formación universitaria en arte es relativamente nueva en nuestro país: hay evidencia de ella desde finales de los años sesenta y comienzos de los setenta. La Academia Superior de Artes de Bogotá, como institución de educación superior, ha tenido que sortear a lo largo de su historia una serie de cambios en sus dinámicas que han interferido directamente sobre los programas que ofrece. Al implementarse la educación teatral como una carrera universitaria formal, todos los énfasis del programa deben acomodarse a las demandas del ministerio y de la universidad a la que pertenecen. El programa de Artes Escénicas de la ASAB es solo un ejemplo de lo vivieron y aún viven muchos de los programas de artes escénicas o dramáticas en el país.

\section{La ASAB}

La formación profesional² en actuación y dirección escénica se constituye en una propuesta curricular a partir del año 1991, cuando se crea la ASAB como una dependencia del Instituto Distrital de Cultura y Turismo (IDCT), integrando las escuelas y academias de formación artística del Distrito, como eran la Academia Musical Emilio Murillo, la Academia Musical Luis A. Calvo, la Escuela de Títeres, Escuela de Cerámica, Escuela de Danza, la Escuela Superior de Teatro Luis Enrique Osorio, la Escuela de Artes Plásticas y la Escuela de Ballet. En las últimas dos décadas esta propuesta curricular ha pasado por distintas recontextualizaciones de los contenidos que orientan la formación de actores y directores escénicos.

Es importante aclarar que el IDCT, como organismo distrital dedicado a la promoción y divulgación cultural, tenía dentro de sus facultades implementar planes de desarrollo de la capacidad artística desde la educación básica primaria hasta la educación básica secundaria y la media vocacional, ofrecida por los planteles distritales anexos a la Secretaria de Educación. La integración de las escuelas y academias de arte adscritas al IDCT —en las cuales durante varios años se formaron y proyectaron talentos artísticos en las distintas disciplinas- en una sola institución encargada de transformar estos programas no formales en programas de educación superior, fue la misión y objetivo principal de la creación de la Academia Superior de Artes de Bogotá.

2 En el ámbito de las artes se presentan dos tipos de profesionales uno validado por su experiencia dentro del circuito artístico y otro validado por las universidades que otorgan títulos profesionales en artes. Es el caso de esta investigación se hace referencia al segundo grupo de profesionales.
Las condiciones surgidas de la Ley 80 de 1980 permitieron fortalecer y desarrollar con éxito la profesionalización en artes, que desde entonces juegan un papel importante dentro de la educación superior colombiana.

La Academia Superior de Artes de Bogotá se configura en consonancia con los lineamientos de la política oficial y desarrolla su compromiso como educadora de artistas en la ciudad proponiendo cinco objetivos: Formar el talento artístico de la ciudad mediante el desarrollo de programas de educación superior; estudiar el patrimonio artístico del país y contribuir en su conservación; contribuir al desarrollo artístico de las comunidades locales y regionales; contribuir a la conformación de redes nacionales e internacionales de investigación y educación artística; y proporcionar asesoría a las organizaciones estatales y privadas encargadas del fomento al desarrollo de actividades artísticas (P.E.I., 1991: 4).

El paso de academia a facultad de arte trajo consigo ciertas resistencias por parte de docentes, estudiantes y administrativos que veían en la fusión con la Universidad Distrital un peligro para la autonomía de la formación de artistas profesionales. Este paso se dio con el acompañamiento de diferentes sectores que representaban a la ASAB y otros tantos que representaban a la Universidad.

Aunque el cambio se venía considerando desde hacía varios años, es en el 2005 (Acuerdo N. 06 de 2005, Consejo Superior) cuando se firma la creación de la Facultad de artes ASAB de la Universidad Distrital Francisco José de Caldas, en adelante (UDFJ). Al tiempo que se realiza el empalme administrativo, surgen inconvenientes que trascendieron lo académico y de lo cual aún existen consecuencias que se evidencian en lo curricular, tal como lo señalan algunos de los entrevistados.

Dentro del programa de Artes Escénicas la tensión se instauró no solamente en nuevas reglamentaciones administrativas (que a la larga traerían implicaciones directas sobre la práctica de la formación de directores y actores escénicos), sino que también se aprovechó la coyuntura para plantear de nuevo el problema entre la orientación de la formación del artista teatral y la del profesional del teatro, que exige la universidad. Así, los tiempos, las dinámicas de la enseñanza y el aprendizaje los espacios, las prioridades, orientaciones y prácticas, eran aspectos que requerían una reflexión a profundidad para reelaborar sus fines y la correspondiente estructura curricular. 


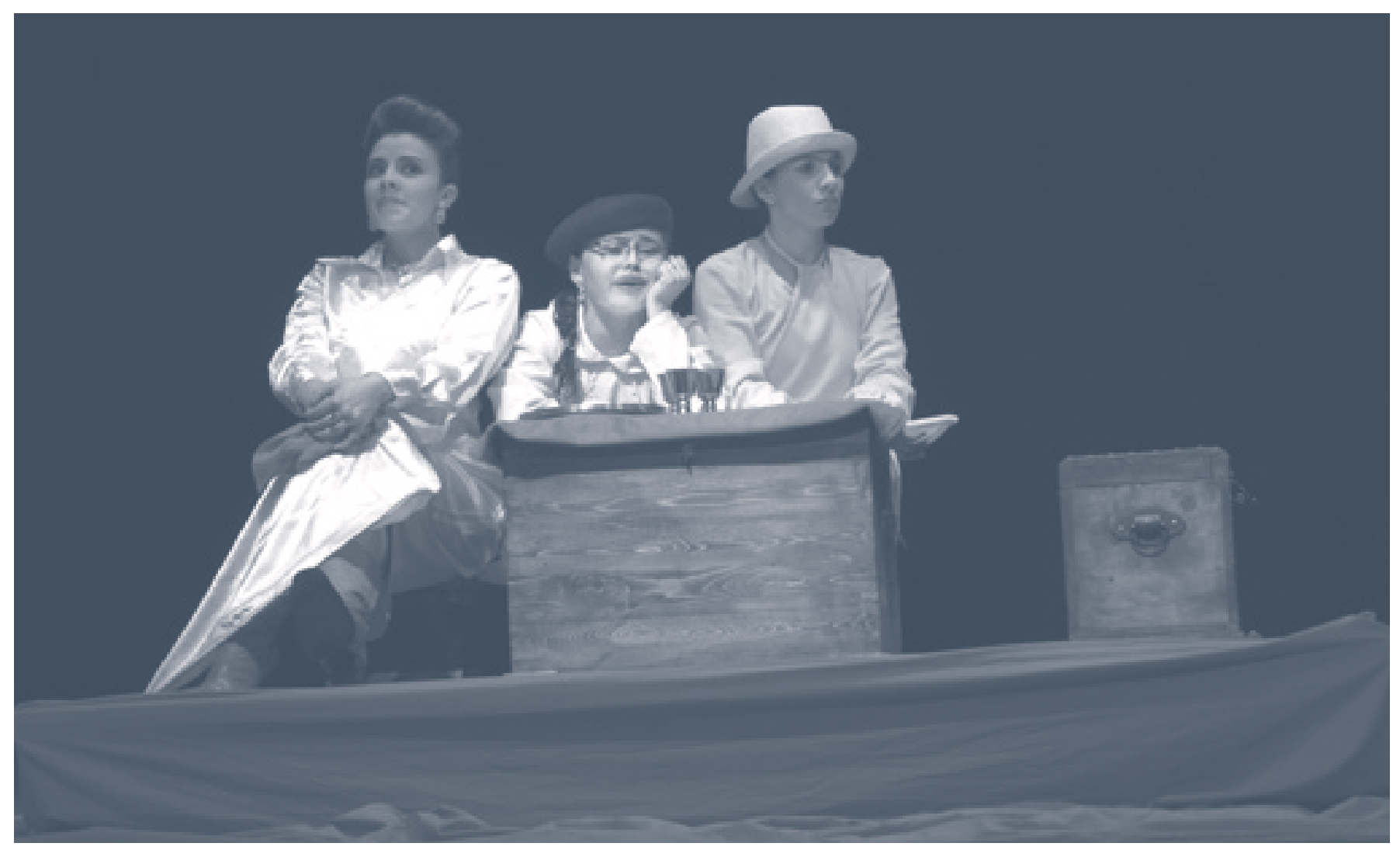

^ A la deriva. Fotografía: Mauricio Rodríguez. 2011

Algunos docentes, administrativos y estudiantes veían en el cambio una posibilidad de salvar la Academia, ya que las políticas culturales del momento impedían que el IDCT continuara manteniendo dentro de sus funciones la responsabilidad de la formación profesional en artes.

Según los relatos de profesores y egresados, la crisis se mantuvo por varios años. Hubo revueltas y procesos de conciliación donde un grupo de docentes señalaba el peligro que corría la ASAB al ser cooptada por la U.D.F.J.C. También se encontraban docentes y administrativos que defendían la fusión. Después de muchas discusiones, en el 2005 se firma la vinculación de la ASAB como Facultad de Artes de la Universidad Francisco José de Caldas.

A partir de este momento los cambios no se hicieron esperar. En el primer periodo académico del 2006, algunos espacios del edificio La Merced ${ }^{3}$ que eran salones fueron destinados a administrativos enviados por la Universidad, como una manera simbólica de demostrar territorialidad. A partir de este año los maestros fueron contratados directamente por la Universidad. Bienestar institucional se hizo presente y con él todos

3 La Merced es el nombre del edificio, donde se ha desarrollado la Academia Superior de Artes de Bogotá, desde 1991. los beneficios para los estudiantes. También llegaron los problemas: algunos empleados fueron despedidos y otros enviados a otras instancias del IDCT; además, el presupuesto asignado por la Universidad no suplía los gastos de la nueva facultad.

Aunque algunos maestros siguen manifestando las desventajas de la fusión, son más los que la defienden, entendiendo que conforme se estaba presentando la situación era muy posible que la Academia Superior de Artes de Bogotá se hubiera atomizado y consecuentemente desaparecido, como ocurrió con la ENAD ${ }^{4}$ en Bogotá y la EPA ${ }^{5}$ en Medellín.

Mientras tanto la Universidad Distrital, a puertas del siglo XX, ya había sorteado una serie de crisis en las

ENAD, Escuela Nacional de Arte Dramático (fundada en 1951 en Bogotá): proyecto académico que se desarrolló en varios países de Latinoamérica y que aún persiste en México, Perú y Argentina, donde se formaron como actores y directores muchos de los grandes artistas escénicos del país. En Bogotá desapareció definitivamente en el año 2003, por razones más administrativas que pedagógicas. 5 La EPA, Escuela Popular de Arte de Medellín fue una de las escuelas de artes que tuvo auge por la década de los 70, como la Escuela Luis Enrique Osorio, hoy ASAB. Allí se ofrecían programas de formación en teatro, danza, música. Al igual que el resto de escuelas desapareció por motivos administrativos. 


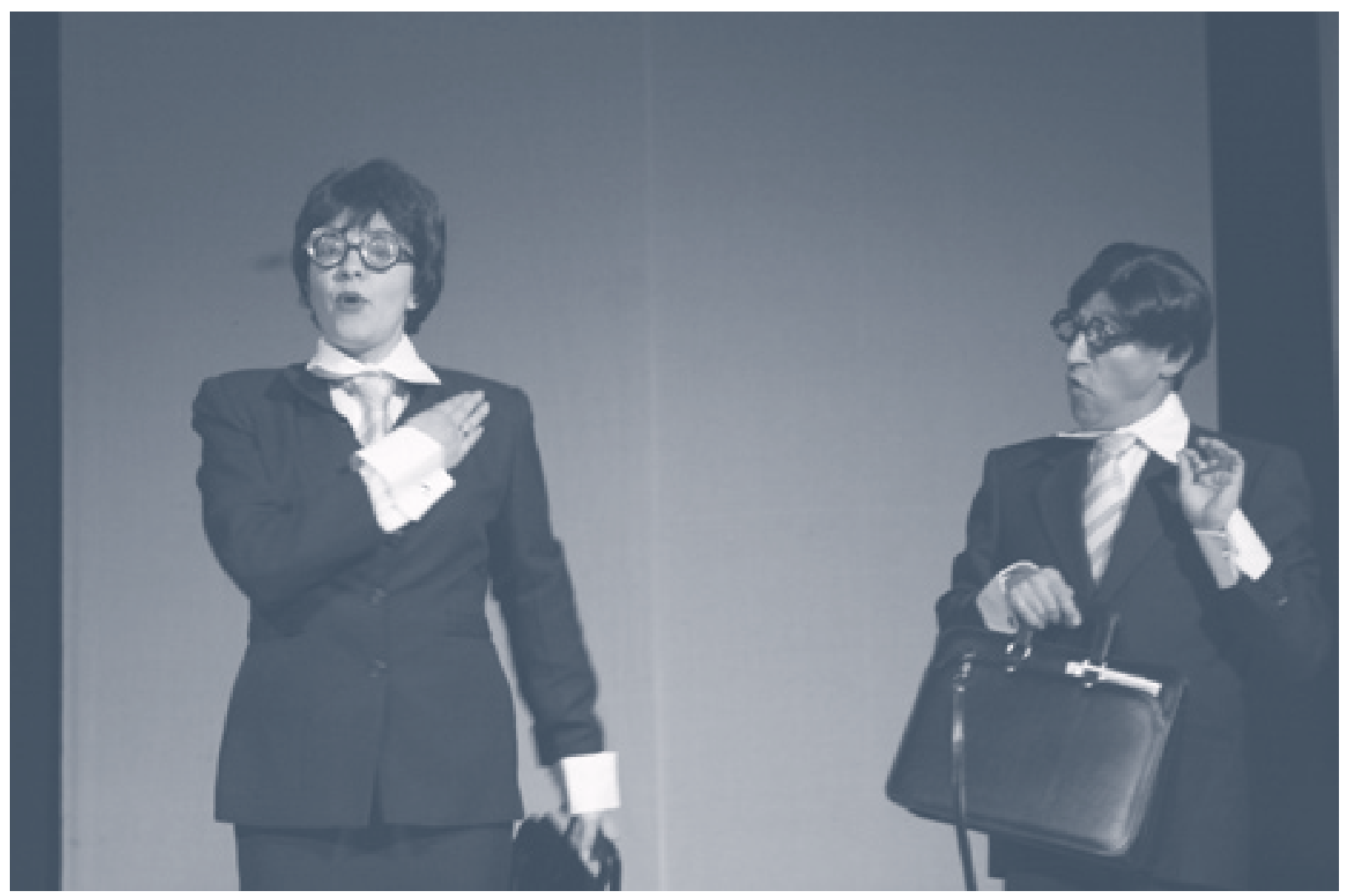

^ Strip Tease, Censura libertad y disentimiento. Fotografía: Mario Rodríguez. 2012

que ponía en juego su destino autónomo y su criterio independiente. Enmarcada en una sociedad capitalista, sus funciones aún siguen evaluándose en consonancia con el "desarrollo" social. Actualmente la Universidad se encuentra respondiendo desde sus fines "a la producción de patrones culturales medios y conocimientos instrumentales, útiles para la formación de mano de obra calificada exigida por el desarrollo capitalista" (De Sousa Santos, 2006: 225).

En una sociedad determinada por las riendas y comportamientos del capital, la universidad pública pasa por una crisis económica causada por el Estado, como bien lo presenta De Sousa: "desde los 90 el gobierno decide reducir su compromiso político con las universidades y con la educación en general, convirtiendo a esta en un bien que siendo público entró automáticamente en una crisis institucional en la defensión por sus principios poco a poco es capturada" (2006: 228). Este fenómeno responde al resultado de un modelo económico que se instauró en algunos países de "el neoliberalismo o globalización universal" (229).

Este proceso de mercantilización no vino solo como salvavidas económico, trajo consigo nomenclaturas provenientes de la industria: palabras como competencias empezaron a instaurarse sin mayores resistencias.
Posteriormente llegaron la exigencias de creditización y acreditación de alta calidad, enmarcando a la Universidad en un escenario de cumplimiento de exigencias para continuar vigente y sobretodo competitiva frente a otras universidades, institutos, escuelas y centros de capacitación como el SENA.

El programa de artes escénicas, como los otros programas de la ASAB, debe adecuarse y responder a las demandas y dinámicas de la Universidad, quien a su vez está en proceso de reestructuración, intentando acelerar sus procesos para adecuarse a las solicitudes del ministerio y de los organismos internacionales de control y vigilancia frente a la educación superior.

\section{$* * *$}

Lo primero que debe hacer el programa es revisar su estructura curricular. Recordemos que frente al currículo y su conceptualización en el campo educativo ha habido diversas posiciones. Desde la perspectiva de Díaz, basada en los desarrollos de Bernstein, el currículo es un producto de la recontextualización. En el campo que nos ocupa implica que se recontextualiza un punto de vista de lo teatral que redefine, sintetiza, propone, reelabora, transforma los elementos que se consideran fundamentales para la formación de 


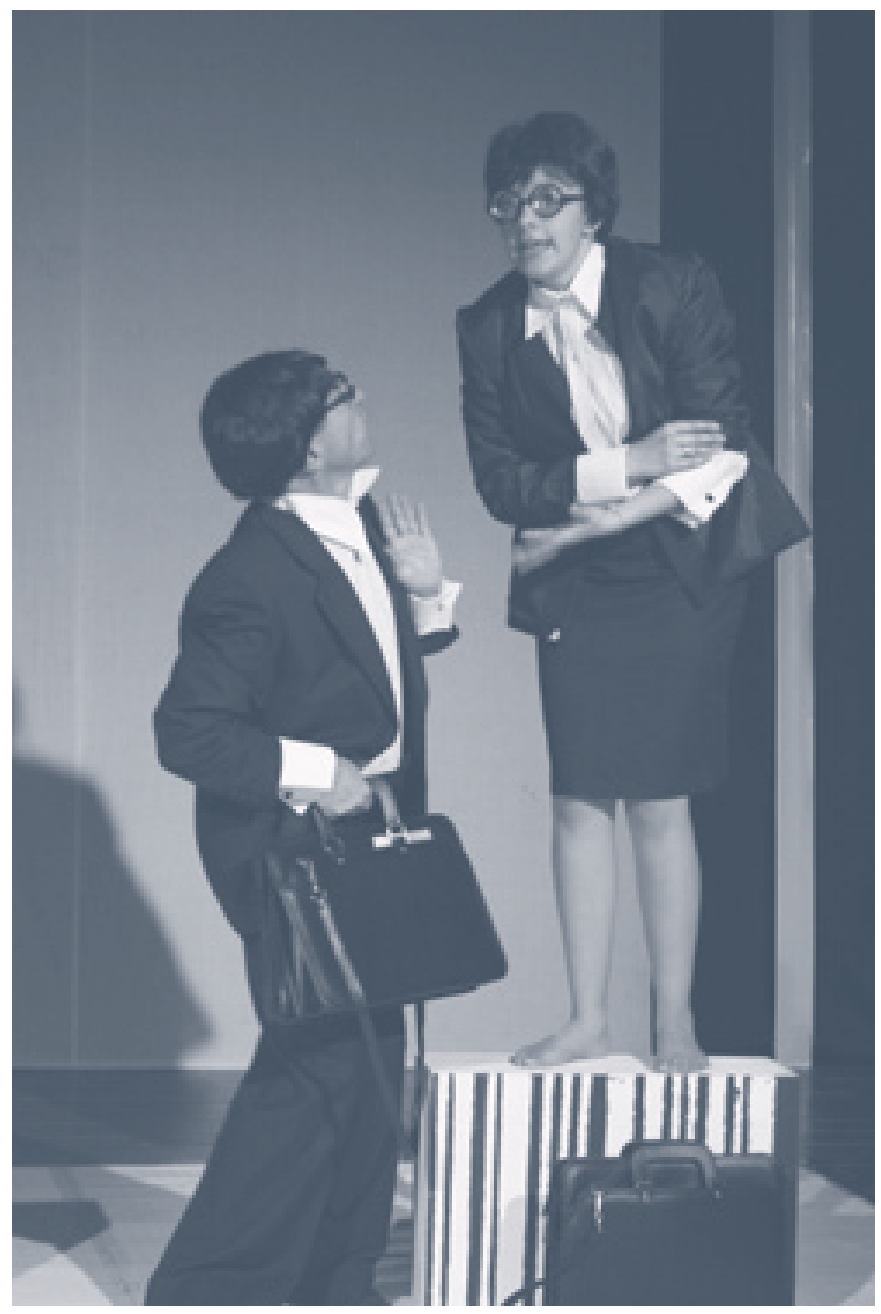

^ Strip Tease, Censura libertad y disentimiento. Fotografía: Mario Rodríguez. 2012

artistas escénicos profesionales, en una coyuntura histórica particular.

A pesar de la diversidad de concepciones que frente al currículo se han señalado, existe una característica común: el currículo obedece a un espacio-tiempo determinado. En palabras de Díaz, “No puede hacerse referencia al currículo en una sociedad en abstracto" (2009: 8). Es decir, todo currículo obedece a un lugar histórico. Lo cual permite entender que el currículo no puede analizarse como un ente aislado sino como un hecho en relación con elementos sociales, culturales y especialmente con las políticas educativas instauradas en cada país.

La selección y organización de contenidos son elementos que obedecen a una estructura curricular y se transforman cada vez que se realiza una modificación, actualización o reforma curricular. Siguiendo a Díaz (2007: 177), “la selección y organización de conocimiento son dos reglas que subyacen a la lógica social de recontextualización del conocimiento". La estructura curricular, según el autor:

Es la columna vertebral de los procesos formativos de una institución. Es la que permite el ordenamiento u organización y relación de los contenidos, las dinámicas y experiencias formativas seleccionadas a partir de las cuales se desarrolla los planes de estudio de los programas académicos de una institución. (2007:68)

En ese orden de ideas cambiar la estructura curricular no corresponde a la acción de quitar y agregar asignaturas, como ocurre en el enfoque de currículo tradicional-agregado (para acomodarse a unas exigencias de número de créditos o porque así lo demanda la universidad); es importante reflexionar sobre las dinámicas que genera una restructuración curricular.

$* * *$

A continuación se contrastan las diferentes propuestas de malla curricular del programa de artes escénicas, donde se evidencia, desde la selección y organización de contenidos de formación, una tendencia por el modelo curricular tradicional-agregado ${ }^{6}$. Vale la pena aclarar que este modelo pedagógico no es exclusivo del programa, es el modelo propuesto por el Estado, al que se han ajustado todos los programas educativos desde la básica y media hasta la superior. Queda la pregunta de si en la autonomía que tienen las instituciones de educación superior un programa $-\mathrm{y}$ más si es un programa de formación artística- no puede optar por otros modelos curriculares como el alternativo-integrado?.

El enfoque o modelo tradicional-agregado se caracteriza por entender la formación como un proceso de transmisión de contenidos que se refiere a un conjunto de técnicas, métodos y operaciones de enseñanza cuyo propósito central, es el de transmitir conocimientos, habilidades y destrezas de una disciplina o profesión; esta tendencia está sustentada en el discurso oficial hegemónico, que insiste en los contenidos, logros y competencias.

El enfoque o modelo alternativo-integrado, se caracteriza por su enfoque crítico e interdisciplinar, donde se le da importancia a la pertinencia social y a la pertinencia académica. Este enfoque considera la formación, como lo señala Díaz (2002), como un conjunto de reglas y principios que generan diferentes clases de prácticas pedagógicas, y reconoce y promueve la autonomía institucional, permitiendo también desarrollos diversos de las competencias y los desempeños en los sujetos. 
Tabla 1

Plan de estudios 1991. Fue el plan con el que inicio el programa de artes escénicas; a partir de él se realizaron las modificaciones posteriores.

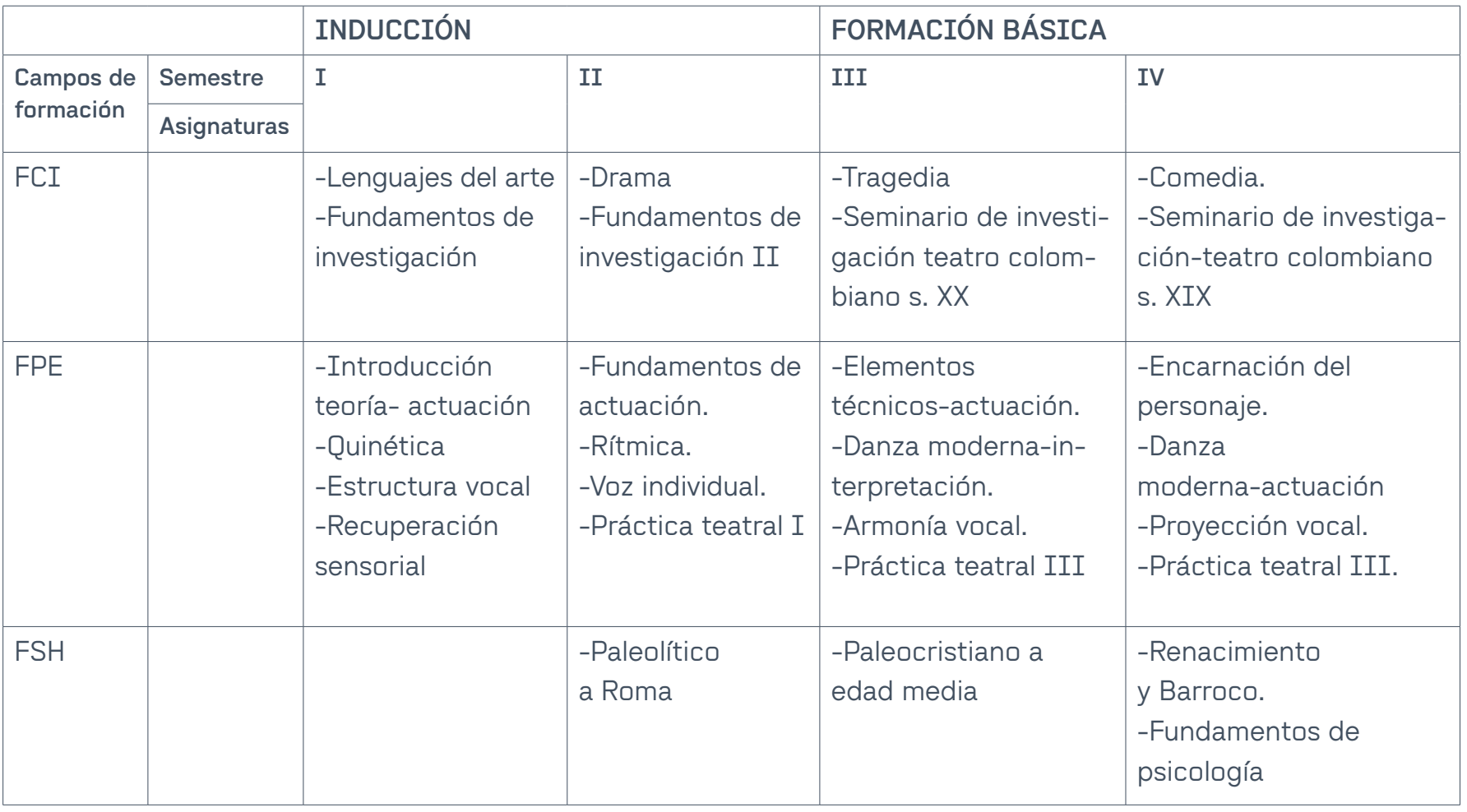

\begin{tabular}{|c|c|c|c|c|c|c|c|}
\hline \multirow{3}{*}{$\begin{array}{l}\text { Campos de } \\
\text { formación }\end{array}$} & \multirow{3}{*}{$\begin{array}{l}\text { Semestre } \\
\text { Asignaturas }\end{array}$} & \multicolumn{2}{|c|}{ FORMACIÓN BÁSICA } & \multicolumn{4}{|c|}{ DESARROLLO PROFESIONAL } \\
\hline & & \multirow[t]{2}{*}{ V } & \multirow[t]{2}{*}{ VI } & \multirow[t]{2}{*}{ VII } & \multirow[t]{2}{*}{ VIII } & \multirow[t]{2}{*}{ IX } & \multirow[t]{2}{*}{$x$} \\
\hline & & & & & & & \\
\hline FCI & & $\begin{array}{l}\text {-Lingüística. } \\
\text {-S.I. teatro colom- } \\
\text { biano s. XVIII. }\end{array}$ & $\begin{array}{l}\text {-Semiología. } \\
\text {-Seminario } \\
\text { investigación } \\
\text { triétnia. }\end{array}$ & -Crítica teatral I & $\begin{array}{l}\text {-Crítica teatral II. } \\
\text {-Empresa teatral II. }\end{array}$ & & \\
\hline FPE & & $\begin{array}{l}\text {-Elementos } \\
\text { construcción del } \\
\text { personaje. } \\
\text {-Danza- nacional. } \\
\text {-Voz- escénica. } \\
\text {-Práctica- teatral IV }\end{array}$ & $\begin{array}{l}\text {-Construcción } \\
\text { del personaje. } \\
\text {-Caracterización } \\
\text { de la danza. } \\
\text {-Caracterización } \\
\text { de la voz. } \\
\text {-Práctica teatral } \\
\text { V }\end{array}$ & $\begin{array}{l}\text {-Empresa teatral I. } \\
\text {-Taller integrado I. } \\
\text {-Montaje I. }\end{array}$ & $\begin{array}{l}\text {-Taller integrado II. } \\
\text {-Montaje II }\end{array}$ & $\begin{array}{l}\text {-Proyecto } \\
\text { artístico }\end{array}$ & $\begin{array}{l}\text {-Trabajo } \\
\text { artístico } \\
\text { de grado }\end{array}$ \\
\hline FSH & & $\begin{array}{l}\text {-s. XVIII a s. XX. } \\
\text { Comportamiento } \\
\text { humano }\end{array}$ & $\begin{array}{l}\text {-Psicología y } \\
\text { sociedad }\end{array}$ & & & & \\
\hline
\end{tabular}

Fuente: Plan elaborado a partir del documento Plan de Estudios de 1991, archivo Secretaría del programa de Artes Escénicas.

Esta fue la malla curricular que se presentó ante el ICFES para que el programa fuera aprobado. No se implementó tal y como aparece aquí; algunos egresados de las cohortes del 96 y 97 manifiestan que no tenían conocimiento de esta malla y aseguran que la dinámica de la relación enseñanza-aprendizaje estaba sustentada en las necesidades particulares del grupo o del montaje. No contaban con horario de clases establecidas; aunque, asistían a clases de voz y cuerpo, la dedicación y conocimientos estaba liderado por el trabajo de montaje.

En 1996 se presenta el siguiente plan de estudios: 
Tabla 2

Organización del Énfasis en actuación, según componentes de formación. (P.E.2 (1996)

\begin{tabular}{|l|l|l|l|l|l|}
\hline Componentes & I Año & II Año & III Año & IV Año & V Año \\
\hline $\begin{array}{l}\text { Fundamentación } \\
\text { profesional }\end{array}$ & $\begin{array}{l}\text {-Expresión corporal } \\
\text {-Expresión vocal } \\
\text {-Técnica especial }\end{array}$ & $\begin{array}{l}\text {-Expresión corporal } \\
\text {-Expresión vocal } \\
\text {-técnica especial } \\
\text {-Apreciación } \\
\text { musical I }\end{array}$ & $\begin{array}{l}\text {-Expresión corporal } \\
\text {-Expresión vocal } \\
\text {-Técnica especial } \\
\text {-Apreciación } \\
\text { musical II }\end{array}$ & $\begin{array}{l}\text {-Expresión corporal } \\
\text {-Expresión vocal } \\
\text {-Técnica especial } \\
\text {-Apreciación } \\
\text { musical III }\end{array}$ \\
\hline $\begin{array}{l}\text { Formación } \\
\text { profesional }\end{array}$ & -Taller de inducción & $\begin{array}{l}\text {-Taller de actuación } \\
\text {-Taller integral }\end{array}$ & $\begin{array}{l}\text {-Taller de actuación } \\
\text {-Taller integral }\end{array}$ & $\begin{array}{l}\text {-Taller de actuación } \\
\text {-Taller integral }\end{array}$ & $\begin{array}{l}\text {-Proyección } \\
\text {-Montaje }\end{array}$ \\
\hline Formación cultural & $\begin{array}{l}\text {-Metodología } \\
\text {-Lenguajes del arte } \\
\text {-Lógica }\end{array}$ & $\begin{array}{l}\text {-Historia del arte I } \\
\text {-Historia del teatro I } \\
\text {-Sicología }\end{array}$ & $\begin{array}{l}\text {-Historia del teatro II } \\
\text {-Historia del arte II } \\
\text {-Patrimonio y cultura }\end{array}$ & $\begin{array}{l}\text {-Historia del teatro III } \\
\text {-Historia del arte III } \\
\text {-Patrimonio cultural II } \\
\text {-Idioma II }\end{array}$ & \\
\hline
\end{tabular}

Nota: Fuente. Documento no publicado. Archivo, Secretaria de currículo programa de artes escénicas, Facultad de Artes ASAB- U.D.F.J.C. 1996.

Este era el plan que se venía desarrollando desde el 1993 pero que solo hasta el 1996 se oficializó. Un aspecto a destacar tiene que ver con el tránsito de campos de formación (científico, investigativo, profesional específico) a componentes de formación, centrados, estos últimos, en la fundamentación profesional, la formación y la cultura. Sin lugar a dudas, estos cambios en la nomenclatura corresponden a las reformas a la ley 30 de 1992, que por la época buscaba una educación pública con gran cobertura y calidad en el desarrollo de sus actividades formativas, implementando la autoevaluación como mecanismo de observación y autorregulación. En 1996 se realiza una reforma a la Ley 30 de 1992 que distingue entre la educación formal (básica, media y superior), la no formal y la informal, lo que le permite a la Academia alejarse cada vez más de lo no formal. Según la reforma, la educación no formal "es la ofrecida con el objeto de completar, actualizar, suplir conocimientos y formar, en aspectos académicos o laborales sin sujeción al sistema de niveles y de grados establecidos, como los descritos para le educación formal". (Ley 30 de 1992, reforma de 1996).

En la propuesta de plan de estudios de 1991 no se tuvo en cuenta los énfasis; sin embargo la división entre actuación y dirección se dio desde la primera cohorte. Para el énfasis de dirección el panorama en la malla curricular del año 1996 presenta la siguiente distribución.

Tabla 3

Organización del Énfasis en dirección, según componentes de formación. (P.E.2 (1996).

\begin{tabular}{|c|c|c|c|c|c|}
\hline Componentes & I Año & II Año & III Año & IV Año & V Año \\
\hline $\begin{array}{l}\text { Fundamentación } \\
\text { profesional }\end{array}$ & $\begin{array}{l}\text {-Expresión corporal } \\
\text {-Expresión vocal } \\
\text {-Técnica especial }\end{array}$ & $\begin{array}{l}\text {-Expresión corporal } \\
\text {-Técnica especial } \\
\text {-Apreciación musical I }\end{array}$ & $\begin{array}{l}\text {-Expresión corporal } \\
\text {-Expresión vocal } \\
\text {-Técnica especial } \\
\text {-Apreciación musical II }\end{array}$ & $\begin{array}{l}\text {-Técnica especial } \\
\text {-Apreciación musical III } \\
\text {-Danza }\end{array}$ & \\
\hline $\begin{array}{l}\text { Formación } \\
\text { profesional }\end{array}$ & -Taller de inducción & $\begin{array}{l}\text {-Taller de dirección } \\
\text {-Taller integral } \\
\text {-S. dirección } \\
\text {-Adaptación I }\end{array}$ & $\begin{array}{l}\text {-Taller de dirección II } \\
\text {-Taller integral } \\
\text {-S. dirección II } \\
\text {-Adaptación II }\end{array}$ & $\begin{array}{l}\text {-Taller de dirección III } \\
\text {-Taller integral III }\end{array}$ & $\begin{array}{l}\text {-Proyección } \\
\text {-Montaje }\end{array}$ \\
\hline Formación cultural & $\begin{array}{l}\text {-Metodología } \\
\text {-Lenguajes del arte } \\
\text {-Lógica }\end{array}$ & $\begin{array}{l}\text {-Historia del arte I } \\
\text {-Historia del teatro I } \\
\text {-Sicología } \\
\text {-Patrimonio cultural } \\
\text {-Idioma }\end{array}$ & $\begin{array}{l}\text {-Historia del teatro II } \\
\text {-Historia del arte II } \\
\text {-Patrimonio cultural I } \\
\text {-Historia de la literatura I } \\
\text {-Escenografía } \\
\text {-Idioma }\end{array}$ & $\begin{array}{l}\text {-Historia del teatro III } \\
\text {-Historia del arte III } \\
\text {-Historia de la literatura II }\end{array}$ & \\
\hline
\end{tabular}

Nota: Fuente. Documento no publicado. Archivo, Secretaria de currículo programa de artes escénicas, Facultad de Artes ASAB- U.D.F.J.C. 1996. 

Sin embargo, al observar los siguientes planes de estudio se encuentra la misma organización. En la propuesta del año 2006, se transpone la idea de campo por la de área, que en últimas termina cumpliendo la misma función de segregación, agrupamiento y límites. entrevistados manifestaron que su práctica de integral era mucho más importante que el seminario o taller de dirección. Este elemento de predilección obedece a la lógica de la formación escénica, que no es la misma lógica para la formación de un ingeniero o un médico.

Tabla 4

Asignaturas suprimidas en el énfasis de Actuación y Dirección (2000)

\begin{tabular}{|c|c|c|c|c|}
\hline año & $\begin{array}{l}\text { E. Actuación, } \\
\text { intensidad horaria } \\
\text { por semana }\end{array}$ & $\begin{array}{l}\text { E. Dirección } \\
\text { intensidad horaria } \\
\text { por semana }\end{array}$ & Materias suprimidas & $\begin{array}{l}\text { Componente al que } \\
\text { pertenecían }\end{array}$ \\
\hline I & 2 & 2 & Metodología & Formación cultural \\
\hline II & 2 & 2 & Lógica & Formación cultural \\
\hline II, III y IV & 1 & 1 & Técnicas especiales & Fundamentación profesional \\
\hline II & 2 & 2 & Sicología & Formación cultural \\
\hline III y IV & 2 & 2 & Patrimonio cultural I y II & Formación cultural \\
\hline III y IV & 4 & 4 & Idioma I y II & Formación cultural \\
\hline I, II y III & 2 & & Apreciación musical & Fundamentación \\
\hline V & 4 & 4 & Proyección & Formación profesional \\
\hline III & & 2 & Escenografía & Fundamentación \\
\hline IV & & 2 & Danza & Fundamentación \\
\hline II y III & & 4 & Seminario de dirección & Formación profesional \\
\hline $\begin{array}{l}\text { Total horas supri- } \\
\text { midas-Actuación }\end{array}$ & 23 & & & \\
\hline $\begin{array}{l}\text { Total horas supri- } \\
\text { midas-Dirección }\end{array}$ & & 29 & & \\
\hline
\end{tabular}

Nota: Fuente sin publicar, archivo Secretaría currículo. Academia Superior de Artes de Bogotá. 2000

Los tiempos de dedicación se regulan desde los mismos principios de organización, la mayor dedicación será para las asignaturas correspondientes al campo o área de formación profesional, porque es la que aparentemente presenta las asignaturas que le permitirán al estudiante su desarrollo específico.

Para el programa de artes escénicas, sin lugar a dudas existe una predilección por las asignaturas que configuran la formación específica; sin embargo, frente al desarrollo de las actividades, las áreas de voz y de entrenamiento que se ubican dentro del campo de formación básica han ganado igual acogida que las áreas de actuación y dirección respectivamente.

Es importante reconocer que en la práctica escénica la jerarquización establecida desde el plan de estudios no siempre es la misma que opera en el desarrollo con los estudiantes. Algunos egresados de dirección
Por eso se insiste en la particularidad de la formación teatral, para este caso.

Los créditos se instauran en el programa de artes escénicas mediante el Acuerdo 009 de septiembre 12 del 2006 y la resolución 035 de septiembre 19 de 2006 del Consejo Académico. Además se señala que "para materializar esta reglamentación se crea mediante la resolución 452 del 30 de noviembre del 2006 desde rectoría, el comité de currículo" (I.A.A., 2011: 162). Este comité, en consonancia con los requerimientos de la Universidad (que se encontraba realizando las gestiones para presentar su postulación al registro calificado de alta calidad) aprueba mediante Acta 014 del 5 de mayo del 2009, los siguientes aspectos:

Autorización para la migración al sistema de créditos a partir de 03-2009. 
Núcleos, componentes y áreas, propuesta de Plan de estudios 2006.

\begin{tabular}{|c|c|c|c|}
\hline \multirow{14}{*}{ NÚCLEOS } & \multirow{8}{*}{ FORMACIÓN BASICA } & COMPONENTES & ÁREAS \\
\hline & & \multirow{4}{*}{$\begin{array}{l}\text { Contextualización y formación } \\
\text { socio-humanística }\end{array}$} & Teorías e historias \\
\hline & & & Humanidades \\
\hline & & & Área común facultad \\
\hline & & & Área común Universidad \\
\hline & & & Técnicas y análisis \\
\hline & & \multirow{2}{*}{ Fundamentación } & Composición \\
\hline & & & \multirow{2}{*}{ Investigación } \\
\hline & \multirow{6}{*}{$\begin{array}{l}\text { FORMACIÓN } \\
\text { PROFESIONAL }\end{array}$} & \multirow{3}{*}{$\begin{array}{l}\text { Formación en el campo de la } \\
\text { profesión }\end{array}$} & \\
\hline & & & Creación Escénica \\
\hline & & & Desarrollo Técnico \\
\hline & & \multirow{3}{*}{$\begin{array}{l}\text { Profundización y } \\
\text { complementación }\end{array}$} & Gestión y producción \\
\hline & & & Informática \\
\hline & & & Electivas \\
\hline
\end{tabular}

Nota: Fuente sin publicar, informe de autoevaluación con fines de acreditación 2011.

- Autorización de tres cátedras institucionales transversales a partir de 03-2009.

- Cátedra Francisco José de Caldas, cátedra de democracia y ciudadanía.

- Cátedra de contexto, una por cada facultad. A(I.A.C., 2011: 162).

El tránsito curricular de horas a créditos trajo consigo dificultades, ya que el número de asignaturas presenciales que desarrollaban los estudiantes no era posible dentro de los 180 créditos permitidos.

Lo que se observa en la Tabla 5 es una evidencia de la acomodación de los nombres: primero campos de formación (1991), luego componentes de formación (1996), y ahora núcleos de formación (2006). Sin lugar a dudas, la modificación más dramática del año 2006 se vivió en el cambio de horas a créditos, ya que no solamente se permitía cambiar un nombre por otro, sino que obligaba al programa a reacomodar sus asignaturas en tiempos concretos, con espacios determinados, especificando las actividades a desarrollar y el tiempo de dedicación tanto del estudiante como del docente.

\section{Impacto}

Con el telón de fondo donde el arte, la educación y la cultura han sido capturados por las nomenclaturas y dinámicas del mercado globalizado es inevitable pensar que un programa de artes escénicas en un país considerado del Tercer Mundo seguirá los mismos derroteros. Sin embargo, es importante resaltar que el programa se ha desarrollado por más de veinte años con la intensión de preservar la esencia de lo teatral por encima de lo productivo.

Esta fortaleza se convierte en debilidad en el crisol de una educación superior enmarcada en las nuevas políticas productivas y de competitividad global. Debido a su adopción tardía de estas dinámicas, que hoy por hoy le cuestan en lo conceptual y en lo propositivo, el programa de artes escénicas, en la última década, ha sufrido una vertiginosa acomodación a ellas.

Es por ello que desde la propuesta curricular del año 1991 hasta la del año 2006 se encuentra un diseño curricular que corresponde al modelo tradicional-agregado, "caracterizado por una relación cerrada de los conocimientos, verticalista, asignaturista, enciclopédico, atomizado, descontextualizado, rígido, ahistórico" (Díaz, 2007: 88), asegurando la jerarquización, el ordenamiento, la selección y distribución de los discursos que deben reproducirse en el campo educativo.

Aunque el programa de artes escénicas busca mantener una orientación específica en la formación teatral, lleva implícita la tendencia inevitable al entrar en el lenguaje de la universidad. Este proceso hace que los procesos se enfoquen en el cumplimiento de exigencias 
en términos de créditos, competencias, flexibilidad, accesibilidad, acreditación, calidad, transversalidad, lo que transforma la selección y organización de los contenidos de formación. Estos empiezan a responder más a estos criterios que a las necesidades específicas de la formación de artistas escénicos, profesionales que se desempeñarán en un mundo donde el arte es un producto para el entretenimiento "en la era del acceso" (Rifkin, 2000).

Queda en el escenario la pregunta: ¿Qué papel desempeñan los artistas escénicos en el país?

El reto del programa de artes escénicas y de los nuevos programas de formación escénica no se limita a la próxima reforma curricular o al desarrollo con fines de acreditación por otros siete años. Los programas no pueden estar de espaldas a esta coyuntura capitalista donde "el capitalismo industrial, está culminando su transición hacia un capitalismo cultural plenamente desarrollado, apropiándose no sólo de los significados de la vida cultural y de las formas de comunicación artísticas que los interpretan, sino también de sus experiencias de vida" (Rifkin, 2000: 195).

Es claro que los programas de artes escénicas deben compaginar dos escenarios: por un lado está la universidad, con sus dinámicas y determinaciones políticas, organizativas y económicas, y por otro lado se encuentra el trabajo teatral inmerso dentro de la libertad de lo artístico. Como proyectos académicos su deber ser es crear los espacios necesarios para que se formen artistas teatrales capaces de transformar, redefinir, recontextualizar su entorno e impactar su contexto.

\section{Referencias}

Antei, G. (1989). «Teatro colombiano: una interpretación», en G. Antei, Las rutas del teatro (págs. 161-174). Bogotá: Universidad Nacional.

(1989). «Viejo teatro y nuevo mundo», en G. Antei, Las rutas del teatro (págs. 175-182). Bogotá: Universidad Nacional.

Arana, T. (2008). La formación teatral universitaria en Colombia, una historia joven no contada. Bogotá: sin publicar.
Aristóteles, y Wilson Rojas (2000). Poética. Bogotá: Wilson Rojas-Editor.

Ariza, P. (1989). «Pequeño diccionario del teatro colombiano», en G. Antei, Las rutas del teatro. Bogotá: Universidad Nacional.

Assad, J. F. (2000). Proyecto modificación curricular. Bogotá.

Caldas, U. D. (2002). Recuperado el 22 de 1 de 2013, de www.udistrital.edu.co

Craig, G. (1999). Principios de dirección escénica. México: Grupo Editorial Gaceta S.A.

De Alba, A. (1.999). Curriculum, crisis, mito y perspectivas. Buenos Aires-Argentina: Miño y Dávila S.R.L.

De Sousa Santos, B. (2006). De la mano de Alicia. Bogotá: Siglo de Hombres Editores.

Díaz, M. (2001). Del discurso pedagógico: problemas críticos. Bogotá- Colombia: Cooperativa Editorial Magisterio.

Díaz, M. (2007). Lectura crítica de la flexibilidad. La educación superior frente al reto de la flexibilidad. Bogotá: Editorial Magisterio.

Educación, M. d. (2004). unesco.org. Consultado en enero de 2013.

Gadamer, H.-G. (1991). La actualidad de lo bello. Barcelona: Paidós.

Hernández Samperio, R. (2006). Metodología de la investigación cuarta edición. Barcelona: Mc Graw Hill.

Lazzarato, M. y. (2004). Capitalismo cognitivo, propiedad intelectual y creación colectiva. Madrid: Traficante de sueños.

López, J. (2001). La de-construcción curricular. Bogotá: Magisterio.

ONU, O. d. (2006). Hoja de ruta para la educación artística: construir capacidades creativas para el siglo XXI. Lisboa.

Ospina, W. (2012). La lámpara maravillosa. Medellín: Mondadori. 
Pardo, J. (1989). «Síntesis de treinta años», en G. Antei, Las rutas del teatro. Bogotá: Universidad Nacional.

Pavis, P. (1990). Diccionario del teatro. Barcelona: Paidós Ibérica S.A.

Programa de Artes escénicas. ASAB. (1991). Plan de estudios, presentado al ICFES Bogotá.

Programa de Artes escénicas - ASAB. (1996). Propuesta modificaciones y ajustes curriculares del programa de artes escénicas. Bogotá.

Programa de Artes escénicas - ASAB. (2000).

Documento modificaciones y ajustes curriculares del programa de artes escénicas. Bogotá.

Programa de Artes escénicas - ASAB. (2011). Informe de autoevaluación con fines de acreditación. Bogotá.

Rifkin, J. (2000). La era del acceso. Barcelona: Paidós.

Tatarkiewicz, W. (2007). Historia de seis ideas, arte, belleza, forma, creatividad, mímesis y experiencia estética. Madrid: Tecnos-Alianza.

Ubersfeld, A. (1998). Semiótica teatral. Madrid:

Ediciones Cátedra S.A.

Universidad Distrital Francisco José de Caldas. (1991).

Plan de estudios, programa de Artes escénicas. Bogotá. 